\title{
Christliche Presse im säkularen Umfeld
}

\author{
von Louis Bosshart*
}

Die Frage, ob Massenmedien Dienstleistungsbetriebe oder Führungsinstrumente seien, bewegt die medienpolitische Diskussion immer wieder. Je nach politischer Ausrichtung der Votanten fallen die Antworten anders aus. Im Geltungsbereich totalitärer Systeme besteht kein Zweifel, daß die Massenmedien wichtige Erziehungsinstanzen sind. Für die liberale Marktwirtschaft ist das Geschäft mit massenmedialen Inhalten ein Erwerbszweig wie andere auch. In der Realität allerdings vermischen sich die unterschiedlichsten Aspekte in erheblichem Maße. Die am Produkt orientierte Auffassung vom Leistungsziel der Massenmedien verliert - mit Ausnahme der hochmotivierten Sympathisanten - relativ rasch ihr Publikum, wenn sie die Bedürfnisse seitens der Nachfrage ignoriert. Die reine Marktorientierung verliert ihre Legitimation, wenn sie aufgrund rein absatzzentrierter Marketingstrategien die Interessen der jeweiligen $\mathrm{Ge}$ sellschaft als Ganzes aus dem Auge verliert.

Für die christliche Presse stellt sich die Frage nach einer produkt- resp. marktorientierten Angebotspolitik in einer ganz speziellen Weise: Auf der einen Seite steht klar der evangelische Missionsauftrag, und es fehlt nicht an kirchlichen Stimmen, die die Massenmedien weltweit als eine Kanzel der katholischen Kirche sehen möchten. Diese Vision läßt sich aber nur in geschlossenen Systemen realisieren, wo die Zielpublika keine Auswahl- und somit Ausweichmöglichkeiten haben. Auf der anderen Seite steht die Leitidee christlicher Toleranz, die auf gegenseitiger Achtung beruht, und es steht auch der offene Markt der Massenkommunikation in vielen Gesellschaften, der unterschiedliche Anbieter zuläßt und verschiedenste Bedürfnisse zu befriedigen erlaubt. Die liberal-demokratische Vorstellung in bezug auf den Leistungsauftrag der Massenmedien setzt - zumindest in den deutschsprachigen Demokratien Europas - eine Selbststeuerung der Gesellschaft mit Hilfe der Massenkommunikation zum Ziel. Eine Regulierung der Gesellschaft durch irgendeine Elite ist also weder gewünscht, noch im Rah. men der gegebenen Marktmechanismen möglich. Wie aber soll die christliche Presse in einer weltlichen Umgebung ihre spezifische Botschaft an das Publikum heranbringen?

Die Antwort wird bereits vorbestimmt durch die Art und Weise, wie in westlichen Demokratien die Massenmedien organisiert werden. In den dualen Mediensystemen der deutschsprachigen Demokratien werden Presseunternehmen im Prinzip privatwirtschaftlich organisiert. Das heißt, daß auch die christliche Presse den Mechanismen des Marktes und des publizistischen Wettbewerbs ausgesetzt ist. Wenn auch in Einzelfällen mehr oder weniger großzügige Subventionen das Überleben einzelner Presseprodukte erleichtern oder gar ermöglichen, wird auch hier immer wieder gefragt, mit welchen Maßnahmen die Marktchancen und damit eben auch die Wirtschaftlichkeit einzelner Titel oder Unternehmen verbessert werden können. Das Institut für Journalistik und Kommunikationswissenschaft der Universität Freiburg (Schweiz) hat in den letzten drei Jahren mehrere Untersuchungen für christliche, resp. katholische Medienunternehmungen durchgeführt, die zeigen sollten, welche Marktlücken (Bedürfnissegmente) für christliches Gedankengut bestehen und welches die $Z$ wänge sind, die von einem offenen Medienmarkt und von Mediennutzungsgewohnheiten auch auf christliche An-

\footnotetext{
${ }^{*}$ Prof. Dr. Louis Bosshart ist Professor für Journalistik und Kommunikationswissenschaft an der Universität Fribourg.
} 
bieter Einfluß haben. Die Resultate - mit Gültigkeit für die Schweiz - waren bisweilen brutal, zumeist aber lehrreich: Das christliche Lesepublikum lebt nicht in einem Vakuum. Es ist voll in eine mehrdimensionale Umwelt integriert. Man kann guten Gewissens der Meinung sein, man wisse, was das christliche Lesepublikum brauche. Das nützt aber alles nichts, wenn man nicht weiß, wo man dieses Publikum abholen muß, wie es reagiert und wie es mit den Informationen umgeht. Letztlich entscheidet nämlich immer das Individuum, welchen Botschaften es sich aussetzen will und wie es diese interpretiert.

\section{Katholische Jugendzeitschriften}

Auch der Markt katholischer Jugendzeitschriften läßt sich nicht vom Preismechanismus zwischen Angebot und Nachfrage abkoppeln und durch andere Instrumente bedürfnisgerecht steuern. Weder kirchlich eingeforderte Zwangsabgaben noch Subventionen können die Verbreitung christlichen Gedankenguts sicherstellen, wenn dies nicht publikumsgerecht geschieht. Die Marktchancen katholischer Jugendzeitschriften müssen also systematisch abgeklärt und in eine Redaktionspolitik integriert werden. Die hier zu referierenden Untersuchungen hatten zum Ziel, Werturteile, Reaktionen und Erwartungen von Jugendlichen unterschiedlicher Altersklassen in bezug auf verschiedene Jugendzeitschriften zu untersuchen. Das Ziel dieser Untersuchungen war immer die Verbesserung der Marktchancen nichtkommerzieller Jugendzeitschriften. Manche in diesem Bereich zu machenden Aussagen werden hart oder provokativ wirken. Sie wurden pointiert formuliert, damit die Problembereiche der Zeitschriften klar werden.

Ganz im Sinne eines harten Forschungsdesigns wurden die Testzeitschriften bewußt mit marktstarken Konkurrenzprodukten konfrontiert und verglichen. Die Gegenüberstellung mit kommerziellen Produkten mag auf den ersten Blick abwegig erscheinen. Die Testzeitschriften verfolgen ja bewußt andere als kommerzielle Ziele, und es soll auch keiner Verkommerzialisierung der katholischen Zeitschriften das Wort geredet werden. Vielmehr soll gezeigt werden, wo man die Jugendlichen aufgrund ihrer Mediennutzungsgewohnheiten abholen muß. Die Leitbilder der Testzeitschriften wurden in verschiedenen Schriftstücken folgendermaßen formuliert:

Zeitschrift A: - „Die Redaktion möchte Buben und Mädchen im Rahmen des christlichen Weltbildes formen und unterhalten, Anleitungen bieten zu sinnvoller Aktivität, religiöse Werte vermitteln, zu sozialem Engagement erziehen. Die Zeitschrift möchte Lebenshilfe anbieten, konfliktfähig machen und eine offene Haltung und Achtung gegenüber der Eigenart anderer Menschen und Völker (Dritte Welt) pflegen."

- „Es konkretisiert die Ziele kirchlicher Medienarbeit. „A sucht die Begegnung mit Schülern und trägt zur öffentlichen Meinungsbildung bei Kindern und Jugendlichen bei. Mit der Verbreitung von , $A^{\prime}$-Meinungen tritt , $A^{\prime}$ 'dem vielerorts feststellbaren Abbau humaner Werte und einseitiger Information sowie passivem Konsum entgegen."

Zeitschrift B: - „B“ will zeitoffen und im Rahmen eines christlichen Weltbildes junge Menschen im Alter von 16 bis 25 Jahren unterhalten und gleichzeitig zu kirchlichem, sozialem und politischem Engagement hinführen. Als Jugendzeitschrift wird „ $\mathrm{B}^{\star}$ schon in der Ausarbeitungsphase von jungen Leuten mitgeprägt. - Die Herausgeber wollen mit „B $\mathrm{B}^{\star}$ zwei Bedürfnisse abdecken:

1. Das Bedürfnis nach einer christlichen Jugendzeitschrift, die aufgreift, was in unserer Welt und Zeit betroffen macht: persönliche Fragen, Umweltfragen, Fragen unserer Gesellschaft, religiöse Fragen. Dazu bietet „B“ zehnmal im Jahr 20 Seiten, zweifarbig mit Schwerpunktthema und ständigen Rubriken, reklamefrei; 
2. das Bedürfnis nach einer Zeitschrift für Interessierte und Engagierte an und in kirchlicher Jugendarbeit $({ } \mathrm{B}$ ) wird auch gelesen von Jugendseelsorgern, Pfarrern und $\mathrm{Er}$ wachsenen, die an Jugendarbeit interessiert sind). Wir wollen nicht bloß beim Beobachten und Feststellen bleiben. Uns ist es wichtig, Einzelne und Gruppen anzuregen, konkret etwas zu tun, zu verändern. Dazu bietet „B“ zehnmal im Jahr (den Innenteil) „B speziell für Jugendgruppen und Junge Gemeinde“: 16 Seiten mit Impulsen, Praktischem, religiöser Animation, Veranstaltungskalender, Symbolfoto...

Seit eineinhalb Jahren wird „B“ auf ap-Umweltschutzpapier gedruckt. Die Herausgeber verstehen dies als einen möglichen Beitrag für eine sinnvolle Nutzung von Rohstoffen.

Zeitschrift C: - „C“ richtet sich an Leser im Primarschulalter, besonders an Zweit- bis Fünft-Klässler. Die Zeitschrift versteht sich als katechetische Unterrichtshilfe. „C" möchte eine offene Haltung und Achtung gegenüber der Eigenart anderer Menschen und Völker fördern, christliche Werte vermitteln und zu sozialem Engagement anleiten. Die jungen Leser sollen mit einem zeitgemäßen Missionsverständnis vertraut gemacht werden.

\subsection{Die Untersuchungen}

Zentraler Untersuchungsbereich war eine Marktstudie. Es wurden an verschiedenen Orten in der Schweiz in verschiedenen Schulklassen die Reaktionen von insgesamt 311 Schülern auf die Testobjekte geprüft. Gleichzeitig wurden die Reaktionen der Testpersonen auf vergleichbare kommerzielle Jugendzeitschriften erhoben. Konkret wurden folgende Untersuchungen durchgeführt:

Tabelle 1: Untersuchungen zur katholischen Jugendpresse in der Schweiz

\begin{tabular}{|l|c|c|c|l|}
\hline Altersklassen & N & Mädchen & Knaben & Methoden \\
\hline 10-11 Jahre & 46 & 20 & 26 & $\begin{array}{l}\text { schriftliche Befragung } \\
\text { Gruppendiskussion }\end{array}$ \\
\hline 10 Jahre & 26 & & & Collage erstellen \\
\hline 13 Jahre & 83 & 47 & 36 & $\begin{array}{l}\text { schriftliche Befragung } \\
\text { Gruppendiskussion }\end{array}$ \\
\hline 14 Jahre & 22 & 9 & 13 & Aufsatz \\
\hline 15-20 Jahre & 182 & 93 & 89 & $\begin{array}{l}\text { schriftliche Befragung } \\
\text { Gruppendiskussion }\end{array}$ \\
\hline 16 Jahre & 23 & 10 & 13 & Aufsatz \\
\hline
\end{tabular}

Der Ablauf bei den Befragungen und Diskussionen war bei allen Altersstufen gleich: Es ging darum, den Bekanntheitsgrad der verschiedenen Produkte zu prüfen, die Lesegewohnheiten zu erheben, die Vorlieben bei der Lektüre in verschiedenen Altersgruppen kennenzulernen und die Vertriebskanäle zu erfassen. Zuerst wurde an die Schulkinder ein allgemein gehaltener Fragebogen zu den genannten Erhebungsbereichen ausgeteilt. Dieser mußte schriftlich ausgefüllt und dann gleich wieder abgegeben werden. Nach dem Ausfüllen der Fragebögen wurde den Testpersonen zuerst ein Testobjekt und etwa zehn Minuten danach ein kommerzielles Vergleichsprodukt abgegeben. Nach weiteren fünf Minuten wurde im Klassenverband unter der Leitung eines Studenten oder einer Studentin eine Diskussion anhand eines Interview-Leitfadens geführt. Dabei wurde spezifisch nach dem Bekanntheitsgrad der beiden zu vergleichenden Zeitschriften gefragt. Die Testpersonen mußten die Aufmachung der jeweiligen Produkte 
beurteilen, ihre Themenpräferenzen zu Protokoll geben und Antwort darauf geben, ob die jeweiligen Zeitschriften Jugendlichen überhaupt etwas bringen. Die Ergebnisse dieser Gruppeninterviews wurden von den Diskussionsleitern teilweise nach Tonbandaufnahmen, teilweise nach Notizen in einem Bericht zusammengefaßt.

\subsection{Problembereich}

Es hat sich gezeigt, daß die Testpersonen über alle Altersklassen sowohl in bezug auf die katholischen Untersuchungszeitschriften als auch auf die kommerziellen Kontrollzeitschrift ähnlich reagierten, und zwar in bezug auf folgende Variablen:

\subsubsection{Aufmachung/Layout}

Die jungen Testpersonen haben ihre Werturteile in bezug auf die einzelnen Zeitschriften in den meisten Fällen direkt mit den Eindrücken von der Aufmachung in Verbindung gebracht. Die Vorzüge der kommerziellen Zeitschriften ( “Bravo“, ,Schweizer Jugend $^{“}$, , pop-rocky“) können folgendermaßen zusammengefaßt werden:

- gute Übersicht, klare Themenstrukturierung

- poppige Farben

- bunte, anregende, abwechslungsreiche Aufmachung

- ansprechendes, marktgängiges Format (A-4).

Daneben nehmen sich die katholischen Blätter ziemlich fade, langweilig, trist und farblos aus. Nachteile, die durch eine professionelle Gestaltung durchaus wettgemacht werden können. Katholizität muß nicht im Gewande eines Aschenbrödels einhergehen. Vor allem ist festzuhalten, daß die Jugendlichen Maß an den kommerziell erfolgreichen Produkten nehmen. Die katholischen Mitbewerber werden daran gemessen. Das Anspruchsniveau der Leser in bezug auf das Erscheinungsbild ist hoch. Auch im Bereich des Standard-Formats setzt die Konkurrenz die Maßstäbe.

\subsubsection{Verteilkanäle, Vertriebsweise}

Die untersuchten katholischen Zeitschriften werden mehrheitlich über Pfarreien (Ministranten) oder Jugendgruppen abgesetzt. Dadurch riskieren sie, in das Presseghetto abzudriften. Die befragten SchülerInnen und Jugendlichen erstehen ihre Zeitschriften vorab am Kiosk oder in Läden. Mit dem Einzelverkauf, oder mit halb-, resp. vierteljährlichen Abonnements werden die kommerziellen resp. Zeitschriften preislich für die Käufer attraktiver, als wenn man den Gesamtkaufpreis auf einmal erlegen muß. Wenn die Kinder ihre Zeitschriften selber und in aller Öffentlichkeit erstehen, werden die Zeitschriften selber stärker öffentlich und so zum Gegenstand kameradschaftlicher Diskussionen und Tauschaktionen. Der vorab innerkirchliche oder vereinsinterne Vertrieb fördert mehr die Absonderung als die Bekanntheit.

\subsubsection{Coin of exchange}

Zeitschriften werden in den jugendlichen Subkulturen zu Diskussionsgegenständen. Man muß sie gelesen haben. Für die katholischen Zeitschriften bedeutet dies, daß auch sie ,Gesprächsstoff liefern sollten. Beliebt sind zudem großformatige Poster sowie Autogrammkarten, die gesammelt und getauscht werden.

\subsubsection{Titel}

Kommerzielle Anbieter lassen die Titel ihrer Zeitschriften verschiedenen Tests unterziehen. Auch hier müßten im vorliegenden Fall die katholischen Zeitschriften von ihren braven und faden Titeln wegkommen.

\subsubsection{Erscheinungsrhytmus}

Der Erscheinungsrhytmus der verschiedenen Zeitschriften trägt viel zur Gewöhnung der Leser bei. Während die Konkurrenzprodukte bis zu einmal wöchentlich erscheinen, erscheinen die Testzeitschriften alle zwei Wochen oder einmal pro Monat. Das ist 
zu wenig. Die Aufmerksamkeit der jugendlichen Leser sollte durch Umfang und Präsenz gefesselt werden.

\subsubsection{Aufwärtsorientierung}

Die untersuchten Jugendzeitschriften stehen nicht nur mit gut gemachten kommerziellen Produkten in Konkurrenz. Die jungen Leute orientieren sich bereits in den ersten Schulklassen an Presseprodukten für Erwachsene. Dadurch werden sie mit teilweise anspruchsvollen Qualitätsstandards bekanntgemacht und empfinden es als Zumutung, wenn sie mit kleinen Formaten, schlechtem Papier, schwächerem Layout und schlichtem Schreibstil bedient werden. Das heißt in der Folge, daß sich Jugendzeitschriften an größeren Altersspektra zu orientieren haben. Die Zeitschrift „Bravo“ beispielsweise ist sowohl den Erstklässlern wie den Zwanzigjährigen bekannt. Hier fand bei den Testzeitschriften eine Zersplitterung der Kräfte und der Mittel statt, die letztlich für die einzelnen Produkte nachteilig sind.

\subsubsection{Themenpräferenzen}

Bei den fur jugendliche Leser attraktiven Themen haben die katholischen Jugendzeitschriften die größte Treffunsicherheit gezeigt: Sie lagen mehrheitlich neben den Publikumsinteressen. Die Inhaltspräferenzen gestalten sich in den einzelnen Altersklassen wie folgt:

Alter 10-12:

- Rätsel, Witze, Krimis

- Poster

- Musik, Filme, Stars

- Sport

- Technik (Eisenbahn, Flugzeuge)

- Tiere

- Reportagen

Alter 13:

- Tiere

- Stars, Schauspieler, Popstars, Sänger

- Sport

- Witze

- Musikszene

- Fremde Länder, Reisen, Völker

- Rätsel

- Filme

Alter 15-20:

- Sport

- Musik, Pop

- Aktuelles

- Allgemeine Informationen

- Umwelt, Tiere, Natur

- Humor, Unterhaltung

- Technik, Wissenschaft

- Kunst, Kultur, Völker

- Medien

Bei den kommerziellen Zeitschriften liegt das inhaltliche Schwergewicht bei den Themen Aufklärung, Sex- und Beziehungsprobleme, Mode, Pop-, Rock-, Musik- und Radioszene, Romane, Kreuzworträtsel, Tests, Horoskop und Witze. Die Themenschwer- 
gewichte der katholischen Zeitschriften finden sich hingegeben bei Weltanschauung, Religion, Politik und fremden Völkern. Dieser Unterschied ist sicher gewollt. Es stellt sich jedoch die Frage, inwieweit dadurch die Jugend tatsächlich angesprochen wird.

Die jugendlichen LeserInnen müssen ja irgendwo abgeholt werden. Schulthemen verweigern sie in der Freizeit die Gefolgschaft. Die attraktiven Themen liegen klar auf der Hand: Sport, Musik, Tiere, Rätsel, Humor, Stars, Information und Unterhaltung. Kirchliche und katholische Themen müßten nicht fehlen. Sie sollten einfach attraktiv, interessant und ohne Schulmeisterei angeboten werden.

\subsection{Diskussion}

Katholische Kinder und Jugendliche wachsen nicht in einem Vakuum auf. Sie sind das Produkt einer vielfältigen Umwelt, ihre Mediennutzungsgewohnheiten werden durch ein professionelles Medienangebot, durch Freizeitverhalten und Peer-Gruppen-Interessen bestimmt. Diese Vorgaben zwingen Anbietern von katholischen Jugendzeitschriften, wenn sie ihr Zielpublikum nicht verfehlen wollen, gewisse Verhaltensweisen auf. Sie müssen sich nach dem bestehenden Markt und seinen Mechanismen richten. Und Sie müssen minimal das jugendliche Publikum dort abholen, wo es ist. Das heißt zusammengefaßt:

- Es ist notwendig, in die wichtigsten Verteilkanäle hineinzukommen, in den Zwischenhandel via Kioske und vor allem in die Tauschkanäle von Freunden, Kollegen und Schulklassen. Das heißt, die entsprechenden Produkte müssen tauschfähig sein und den jugendlichen Subkulturen entsprechen.

- Der Wettbewerb im publizistischen Bereich wird von den Marktleadern bestimmt, professionell präsentierte Produkte bestimmen die Wahrnehmungsmuster des jugendlichen Publikums und dessen qualitative Ansprüche. Es geht also darum, die Zeichensysteme (Sprache, Bilder, Farben) unserer Zeit zu kennen und zu verwenden. Die Werbung ist sich der Freiwilligkeit der Medienzuwendung bewußt und schaff klar bestimmte Anreize. Christliche Botschaften bedürfen ebenfalls einer attraktiven Aufmachung, wenn sie überhaupt wahrgenommen werden sollen.

- Die jugendlichen Themen sind allgemein bekannt. Es handelt sich vorab um Inhalte aus der Popkultur (Stars, Idole), aus dem Sport, aus der Umwelt, aus persönlichen Anliegen (Freizeitaktivitäten, Drogen, Sexualität, zwischenmenschliche Beziehungen) und aus Spielstoff für den Kopf (Rätsel, Witze, Krimis). Die Themenpräferenzen der jugendlichen Leser korrespondieren mit Freizeitbeschäftigungen und Inhalten von Peer-Gruppen-Diskussionen. Wer Bescheid weiß, ist 'in'. Wer bei den Jugendlichen 'in' sein will, muß entsprechende Angebote machen. Man mag dem entgegenhalten, daß katholische Jugendzeitschriften ganz andere Ziele hätten. Dazu bleibt nun aber zu bedenken, daß die jugendlichen Leitthemen, wie Popkultur, Sport, Umwelt, zwischenmenschliche Beziehungen - um Gotteswillen! - ausreichend Anlaß zu einer Behandlung in christlichem Sinne geben! Die jugendlichen Leser suchen Information, Anregungen, Problemlösungen, Lebenshilfe, Ratgeber - aber keine Lehrer und Belehrungen. Darin sind sie den Erwachsenen gleich. Die Wirkungsforschung weiß seit geraumer Zeit, daß es die Leute nicht gerne haben, wenn man ihnen direkt sagt, was sie zu tun und zu denken haben.

- Die Kleinen folgen noch in anderen Bereichen den Spuren der Großen; Sie orientieren sich an deren Medien. Bereits in den ersten Schulklassen lesen Jugendliche Publikumszeitschriften. Dann folgen Tageszeitungen, und mit etwa 17 Jahren wenden sich die jungen Leute 'Special-interest'-Zeitschriften, politischen Wochenmagazinen und nunmehr vollends den Tageszeitungen zu. Katholische Zeitschriften sollten also - 
wenn sie ein ausreichendes Marktpotential vorfinden wollen - ein ausreichendes Altersspektrum ins Auge fassen. Und sie dürfen durchaus Ansprüche stellen.

Die Ergebnisse der Untersuchungen über die Marktchancen der katholischen Jugendzeitschriften können in wenigen Sätzen zusammengefaßt werden: Katholische Jugendzeitschriften zielen am allgemeinen Interesse vorbei, wenn sie keine Rücksicht auf die jugendlichen, situationsbedingten Bedürfnisse nehmen. Abholen ist im Hinblick auf erfolgreiche Kommunikation ebenso wichtig wie der Inhalt. Jugendliche wollen nicht an die Schule erinnert werden. Ein Massenmedium als Kanzel geht von falschen Vorstellungen über das Publikumsverhalten aus. Katholische Jugendzeitschriften sollten Fermente sein. Wenn sie als das Salz der Erde wirken wollen, müssen sie sich mit ,erdigen' Themen befassen, das heißt, sie müssen die christliche Botschaft in einen optimalen, publikumsgerechten Angebots-Mix einbringen.

\section{Christliche Meinungspresse}

Der Bedarf an weltlicher Information auf der Basis christlichen Gedankengutes konnte auch bei erwachsenen Lesern festgestellt werden. Das Institut für Journalistik und Kommunikationswissenschaft der Universität Freiburg (Schweiz) hat in den letzten drei Jahren für insgesamt fünf Tageszeitungen Leserschaftsanalysen durchgeführt. Dabei ging es in allen Fällen darum, den bestehenden Markt der christlichen Meinungspresse zu überprüfen und Marktnischen für die christliche Meinungspresse zu erforschen. Die folgende Tabelle weist die verschiedenen Untersuchungen aus.

Tabelle 2: Leserschaftsbefragungen zur christlichen Meinungspresse

\begin{tabular}{|l|c|l|l|}
\hline Zeitung & Auflage & Methode & N \\
\hline Die Ostschweiz & 29.000 & schriftliche Befragung & 259 \\
\hline Vaterland & 49.000 & schriftliche Befragung & 306 \\
\hline Nordwestschweiz & 14.500 & schriftliche Befragung & 500 \\
\hline Aargauer Volksblatt & 9.200 & schriftliche Befragung & 197 \\
\hline Freiburger Nachrichten & 13.680 & $\begin{array}{l}\text { telephonische Interviews } \\
\text { schriftliche Befragung }\end{array}$ & $\begin{array}{l}770 \\
915\end{array}$ \\
\hline
\end{tabular}

Bei den untersuchten Zeitungen handelt es sich ausschließlich um Regionalzeitungen, die vorab katholische Regionen abdecken. Der Anteil der Katholiken bei den Antwortenden variiert zwischen 70 und 97 Prozent. Beim übrigen Anteil handelt es sich mit einer klaren Mehrheit um Protestanten.

\subsection{Themenpräferenzen}

Bereits die Frage nach den bevorzugten Themen zeigte klar, daß katholische Leser in ihren Tageszeitungen Informationen zum Alltag und zum persönlichen Lebensvollzug suchen:

- Lokalnachrichten (Gemeinde)

- Kantonalnachrichten

- Regionalnachrichten (Bezirk)

- Wirtschaftsnachrichten

- Inlandsnachrichten

- Auslandsnachrichten

- Sport

- Todesanzeigen

- Kommentare 
- Veranstaltungskalender

- Bilder

- Leserbriefe

Beiträge zu Kirche und Religion finden ein ausgesprochen schwaches Echo. Das will aber nicht heißen, daß christliches Gedankengut keine Nachfrage findet.

\subsection{Charakterisierung der Zeitungen}

Konkret beurteilen konnten die Befragten ihre Zeitung, indem sie angeben konnten, ob gewisse Merkmale, mit denen üblicherweise Printmedien qualifiziert werden, auf die jeweilige Zeitung zutreffen oder nicht. Als Merkmale wurden vorgeschlagen:

glaubwürdig, aktuell, vielseitig, meinungsbildend, hohes Niveau, sachlich, unterhaltsam, umfassend, objektiv. Diese Merkmale (außer unterhaltsam) sind neben anderen Faktoren wichtige Nachfragedeterminanten der Massenmedien überhaupt. Eine hohe Bejahungsquote kann auf eine hohe Akzeptanz des entsprechenden Mediums hinweisen. Die Ergebnisse der hier referierten Befragungen fielen gut aus: Sie weisen auf ein großes Vertrauen der Leser gegenüber der abonnierten Zeitung hin: Von der Mehrheit als zutreffend empfunden, werden die Merkmale, glaubwürdig ' und ,sachlich'. Viele interessierte Leser bejahen ebenfalls die Merkmale ,aktuell', ,vielseitig' und ,objektiv'. Nur von einem kleinen Teil der interessierten Leser als zutreffend erachtet werden die Merkmale ,umfassend', ,unterhaltsam', ,hohes Niveau' und ,meinungsbildend'. Die relativ schwache Befürwortung von ,umfassend ' und ,meinungsbildend' ist recht ungewöhnlich, zumal gerade mit diesen Merkmalen die Zeitung von anderen Massenmedien (Radio, TV) unterschieden wird.

Um festzustellen, ob semantische Gemeinsamkeiten der verschiedenen vorgegebenen Merkmale aufgrund von Korrelationen mit Faktoren beschrieben werden können, wurden die Daten einer Faktorenanalyse unterworfen. Das Verfahren extrahierte drei Faktoren, die 51 Prozent der Varianz erklären.

Faktor 1: wahrheitsgetreu: objektiv $(0,69)$, glaubwürdig $(0,64)$, sachlich $(0,75)$

Faktor 2: abwechslungsreich: aktuell $(0,61)$, vielseitig $(0,73)$, unterhaltsam $(0,42)$, umfassend $(0,61)$

Faktor 3: intelligent: meinungsbildend $(0,77)$, hohes Niveau $(0,67)$, unterhaltsam $(0,51)$

Männer und Frauen unterscheiden sich in der Charakterisierung der christlichen Meinungspresse lediglich in Bezug der Merkmale ,sachlich' und ,objektiv'. Mehr Männer als Frauen finden diese beiden Adjektive als für ihre Zeitung zutreffend. Unterschiedliche Meinungen vertreten die Altersgruppen bei den Merkmalen ,glaubwürdig', ,meinungsbildend" und ,hohes Niveau'. Mit zunehmendem Alter steigt die Tendenz, die Zeitungen mit diesen positiven Merkmalen zu verbinden.

\subsection{Erwartungen}

Hinweise für das redaktionelle Marketing kann die Antwort auf die Frage liefern, was die interessierte Leserschaft denn überhaupt von einer Zeitung erwartet. Im Gegensatz zu den Fragen über die Charakterisierung verzichtet man hier auf Antwortvorgaben, um das Denken der Beantworter nicht in vorgegebene Richtungen zu lenken. Um die Antworten statistisch auszuwerten, wurden diese in einem ersten Durchlauf kategorisiert. Die überraschenderweise vielen Antworten (2564) ließen sich in sieben Kategorien gliedern: christlich, wahr/seriös, sozial/solidarisch, klare Linie, neutral, kritisch und keine Boulevardzeitung. Die folgende Tabelle beschreibt die Kategorien und gibt die entsprechende Zahl der Nennungen an: 
Tabelle 3: Kategorien der christlichen Meinungspresse

\begin{tabular}{|l|c|c|}
\hline Kategorie & Anzahl der Nennungen & Prozent \\
\hline christlich & 409 & 30 \\
wahr, seriös & 191 & 14 \\
sozial, solidarisch & 190 & 14 \\
kein Boulevard & 171 & 12 \\
klare Linie & 132 & 10 \\
neutral & 120 & 9 \\
kritisch & 67 & 5 \\
religiöse Anlässe & 54 & 4 \\
papsttreu & 23 & 2 \\
einfach, konkret & 21 & 2 \\
\hline
\end{tabular}

Die klare Linie, wahrheitsgetreue Berichterstattung ohne Boulevardisierung und die christlich-soziale Haltung sind die am meisten genannten Erwartungen der Leserschaft der christlichen Meinungspresse.

Interessanterweise kann man bei diesen Antworten feststellen, daß journalistischethische und auch allgemein-ethische Haltung erwartet werden. Nur mit wenigen Ausnahmen werden Identifikationen mit Personen (Papst) oder Institutionen (Kirche) verlangt. Überhaupt nie angesprochen werden katholische Parteien oder Politiker.

Wenn auch kirchliche oder religiöse Themen nicht zuoberst auf der Wunschliste katholischer Leser stehen, so schätzen diese doch eine Berichterstattung über weltliche Themen, die ,christlich', wahr, seriös, sozial und solidarisch sowie einer klaren Linie verpflichtet sind.

Eine gewisse Analogie zu den Ergebnissen aus den Untersuchungen zur Jugendpresse ist ganz klar feststellbar: Katholische Erwachsene suchen in ihren Zeitungen nicht primär Informationen über Kirche und Religion, sie schätzen jedoch in hohem Maße eine klare Linie auf der Basis christlicher Weltanschauung. Eine Tageszeitung zu einer Kanzel umfunktionieren zu wollen, würde auch hier bedeuten, daß an den Publikumsbedürfnissen vorbeigepredigt, vorbeiproduziert würde. Das Bild vom christlichen Salz in weltlichen Themen ist auch hier angebracht. Für die Rezipienten ist es wichtig zu wissen, daß ein weltliches Thema auf der Basis einer klaren christlichen und sozialen Weltanschauung behandelt und beurteilt wird. Seriosität und Wahrheit haben eindeutig den Vorrang. Der lesende Erwachsene sucht wie der Jugendliche Informationen, um seine Probleme befriedigend lösen zu können.

\section{Die KIPA}

Nach Artikel 2 der Statuten der Katholischen Internationalen Presse-Agentur verfolgt diese folgende Ziele: „Zweck der Genossenschaft ist das Sammeln, Bearbeiten und Verbreiten von Informationen, Kommentaren und Publikationen über die römischkatholische Kirche, die übrigen Kirchen und Religionsgemeinschaften und über alles, was diese betrift. Dieser Z weck wird erreicht durch den Betrieb einer Nachrichtenagentur."

Agenturen erfüllen im allgemeinen in ihrer Position zwischen Ereignis und Massenmedien drei spezifische Funktionen:

a) Sie suchen in der Welt nach Ereignissen und wählen aus der Realität nach gewissen Selektionskriterien Ausschnitte aus (Gatekeeperfunktion). 
b) Sie transformieren die selektierten Beobachtungen in Nachrichten (Verfasserfunktion).

c) Sie übermitteln die Nachrichten den Redaktionen der Massenmedien, welche diese unter Umständen an ihre Rezipientenschaft weiterverbreiten (Medienanbieterfunktion). Qualitätskriterien einer Agentur sind Aktualität und Wahrheitstreue. Im Gegensatz zu einem Pressedienst liegt die Stärke der Agenturberichterstattung in der kurzen, auf die "Facts“ konzentrierten publizistischen Stilformen der Nachricht (oder Meldung).

Die KIPA hebt sich von allgemeinen Agenturen dadurch ab, daß sie sich in thematischer Hinsicht spezialisiert hat. Sie wählt aus dem Gesamt der möglichen Ereignisse religiöse Ereignisse der katholischen Kirche, aber auch der anderen Kirchen und Religionsgemeinschaften aus und bietet diese den Redaktionen an. Als weitere Schwerpunkte bezeichnet sie die Ökumene, Menschenrechte, Ethik, Frieden, Jugend, Ausländer, Umwelt, Mission, Entwicklung und Kultur.

3.1. Erwartungen und Abonnenten

Bei einer derartigen Ausgangslage steht die Annahme nahe, daß Informationen über religiöse Ereignisse der katholischen Kirche, daß Glaubensfragen, Verkündigung einem Bedürfnis der Abnehmer entsprechen. Dies triff aber nur teilweise zu, nur bei sogenannten Endverbrauchern, das heißt bei kirchlichen Institutionen (Ordensgemeinschaften, kirchlichen Stellen) und Einzelpersonen (Bischöfen, Pfarrherren). Die eigentliche Zielgruppe der KIPA - die Massenmedien - hegt hingegen ganz andere Erwartungen. Während kirchliche Abnehmer des KIPA-Dienstes anläßlich einer Befragung mit den inhaltlichen Schwergewichten der KIPA durchaus zufrieden sind, erwarten Medienvertreter (Multiplikatoren) mehr kritische Distanz, mehr Inlandsnachrichten und weniger Hofberichterstattung.

Zitate:

- Mehr (v.a. auch kritische) Beiträge aus der Schweiz, weniger Banalitäten aus dem Vatikan und von den Papstreisen. Ob die Bischöfe Polens zu mehr Enthaltsamkeit aufrufen, ist unseren Schweizer Lesern wirklich egal.

- Nicht so viele Nachrichten aus Rom, mehr aus der katholischen Kirche der Welt.

- Mehr Objektivität, weniger Verherrlichung der eigenen Religion.

- Größere inhaltliche Unabhängigkeit gegenüber kirchlicher Hierarchie, z.B. kritischere Berichterstattung aus Rom, kein Hofjournalismus.

- Größere Vielfalt der Weltkirche, weniger vatikanischer Hof, auch kritische Stimmen zu kirchlichen Vorgängen.

- Mehr schweizerische Nachrichten.

- Vermehrt religiös-kirchliche Neuaufbrüche, neue Formen von religiösen Lehren und Glauben.

Die Disparität zwischen Klerikern und Journalisten findet eine Parallele bei der Frage nach den gewünschten Textformen. Meldungen/Kurzberichte, Reportagen, Kommentare und andere Meinungsstilformen werden nach Ansicht der Befragten von der KIPA zu wenig benutzt und von fast der Hälfte der Abonnenten vermehrt gewünscht. Während Redakteure und Korrespondenten für einen Informationsdienst, für Radio/ TV, für eine Presseagentur und auch die Mitarbeiter eines bischöflichen Ordinariats vermehrt Meldungen und Kurzberichte benötigen, wünschen sich Redakteure und Korrespondenten für eine Zeitschrift/Zeitung und Mitarbeiter einer kirchlichen Stelle eher Reportagen, Berichte, Kommentare und andere Meinungsstilformen. Für die audiovisuellen Medien Radio/TV, für die Presseagenturen und Informationsdienste zählen also vor allem Meldungen und Kurzberichte, Zeitungen und Zeitschriften verlangen vor allem Hintergrundinformationen, letztlich also Eigenleistungen. 


\subsection{Potentielle Abonnenten}

Die markanten Unterschiede zwischen den Interessen und Erwartungen kirchlicher Personen und Journalisten wurden auch bei einer Befragung potentieller Abnehmer des KIPA-Dienstes deutlich. Die Inhalts-Präferenzen der befragten Kleriker zeigen auch hier eine klare Tendenz zu Informationen über Ereignisse der katholischen Kirche. Dazu kommen noch Themen wie Glaubensfragen, Ökumene, Mission und Ethik. Wenig Nachfrage besteht hingegen bei den Themen Umweltprobleme, Frieden, Ausländerfragen und Kultur. Die befragten Kleriker würden also vorab Informationen aus dem klassischen kirchlichen Bereich erwarten. In diesem Bereich wünschten sich die Kleriker vorab detaillierte Hintergrundinformationen und Kommentare, oder anders formuliert, Analysen und Interpretationshilfen. Während die Kleriker also von einer kirchlichen Presseagentur vor allem mit Informationen über klassische kirchliche Bereiche beliefert werden wollen, wollen die Medien dies gerade nicht. Einzig die Themen Ökumene und Ethik sehen sich einer einigermaßen ausgewiesenen Nachfrage gegenüber. Die Erwartungen in bezug auf die thematischen Angebote zeigen klar folgende Spitzenreiter:

- Umwelt

- Frieden

- Jugend

- Menschenrechte

- Ausländerfragen

(stets aus kirchlicher Sicht)

Die Negativliste führt folgende Themen auf:

- Ereignisse anderer Kirchen

- Mission

- Entwicklung

- Ereignisse der katholischen Kirche

Die befragten Journalisten erwarten also von der KIPA vorab die Behandlung säkularer Themen aus kirchlicher Sicht. Es muß hier noch ganz klar angemerkt werden, daß diese Erwartungen einem bestimmten Marktsegment entsprechen, nämlich den nichtkatholischen, bürgerlichen Zeitungen. Die Stoßrichtung ist ziemlich klar: Die weltlichen Medien wollen vor allen Dingen wissen, was die katholische Kirche zu den groBen Problemen unserer Zeit zu sagen hat (vgl. dazu etwa die Diskussion um die Krankheit AIDS). Aus diesem Grunde wurden denn auch die Stilformen Kommentar und Interview von dieser potentiellen Kundengruppe am häufigsten gewünscht. Im Vergleich zu den Klerikern wollen Journalisten dann aber weit eher Meldungen und Nachrichten denn Reportagen und Berichte. Die folgende Tabelle faßt die Erwartungen verschiedener Abnehmerkategorien zusammen: 
Tabelle 4: Erwartungen verschiedener Abnehmerkategorien in bezug auf die KIPAProdukte

\begin{tabular}{|c|c|c|c|c|}
\hline \multicolumn{2}{|c|}{ Nachfrage $\quad$ Erwartungen } & Themen & Textformen & Bilder \\
\hline \multirow{5}{*}{ 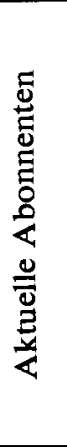 } & $\begin{array}{l}\text { Zeitungen/Zeitschriften } \\
\text { (säkulär) }\end{array}$ & \multirow{5}{*}{$\begin{array}{l}\text { Jugend } \\
\text { Umwelt } \\
\text { Kultur } \\
\text { Ethik } \\
\text { Glaubens- } \\
\text { fragen }\end{array}$} & $\begin{array}{l}\text { Reportagen, Berichte, } \\
\text { Kommentare }\end{array}$ & ja \\
\hline & Radio/TV & & $\begin{array}{l}\text { Meldungen } \\
\text { Kurzberichte }\end{array}$ & nein \\
\hline & $\begin{array}{l}\text { Zeitungen/Zeitschriften } \\
\text { (religiös) }\end{array}$ & & & ja \\
\hline & $\begin{array}{l}\text { Agenturen/Info Dienste } \\
\text { (religiös) }\end{array}$ & & $\begin{array}{l}\text { Meldungen } \\
\text { Kurzberichte }\end{array}$ & nein \\
\hline & $\begin{array}{l}\text { Endverbraucher } \\
\text { (katholisch) }\end{array}$ & & & \\
\hline \multirow{2}{*}{ 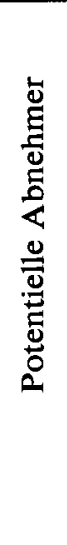 } & $\begin{array}{l}\text { Endverbraucher } \\
\text { (Kleriker) }\end{array}$ & $\begin{array}{l}\text { Info über ka- } \\
\text { tholische Kir- } \\
\text { che; Glaubens- } \\
\text { fragen, Oeku- } \\
\text { mene, Mission, } \\
\text { Ethik }\end{array}$ & $\begin{array}{l}\text { Kommentare } \\
\text { Analysen, Hinter- } \\
\text { grundinformationen }\end{array}$ & \\
\hline & $\begin{array}{l}\text { Journalisten nicht- } \\
\text { katholischer Medien }\end{array}$ & $\begin{array}{l}\text { Umwelt, Friede, } \\
\text { Jugend, Men- } \\
\text { schenrechte, } \\
\text { Info über säku- } \\
\text { läre Themen } \\
\text { aus kirchlicher } \\
\text { Sicht, Ethik, } \\
\text { Oekumene }\end{array}$ & $\begin{array}{l}\text { Kommentare } \\
\text { Interviews } \\
\text { Meldungen } \\
\text { Nachrichten }\end{array}$ & \\
\hline
\end{tabular}

\subsection{Resultate}

Analog zu den Lokalzeitungen findet sich bei der KIPA eine große Bedeutung der überblickbaren Ereignisse. Der internationalen Ausrichtung zum Trotz, werden von der KIPA starke lokale, regionale und nationale Bezüge erwartet. Der Berichterstattung über Ereignisse in Rom wird die Kirche der Schweiz vorgezogen.

Erste Aufgabe einer Nachrichtenagentur ist die Vermittlung von Informationen. Von der KIPA werden als weltanschaulich ausgerichteter Institution aber noch zusätzliche Dienstleistungen erwartet: Sie soll einerseits theologische Themen und Fragestellungen allgemeinverständ lich umsetzen, Interpretationshilfe leisten und Hintergründe recherchieren. Andererseits soll sie die Themen unserer Zeit im Lichte christlicher Ethik behandeln.

Als spezialisierte Agentur muß die KIPA die theologische Gewichtung der Themen zuhanden der Massenmedien vornehmen und eine Auswahl treffen. Vertreter der Massenmedien - katholische wie nicht-katholische - haben in bezug auf ihre Themenpräfe- 
renzen klare Aussagen gemacht. Sie erwarten von der KIPA Informationen zu folgenden Themenbereichen:

- Umwelt

- Jugend

- Frieden

- Kultur

- Menschenrechte und

- Ausländerfragen

Hier handelt es sich eindeutig um zentrale Probleme der Gegenwart, die die Medien im Lichte der katholischen Lehre behandelt sehen wollen. Bei den katholischen Medien stehen zudem Glaubensfragen auf der Themenliste. Ethik und Ökumene sind für alle Medien von höchstem Interesse. Die Behandlung verschiedener Fragen aus ethischer Sicht entspricht in einer Zeit, in der viele nicht mehr wissen, was gut und was schlecht ist, einem großen Bedürfnis. Ethik hilft, die allgemeine Orientierungslosigkeit zu vermindern.

\section{Befunde}

Die Ergebnisse der hier vorgetragenen Studien bestätigen aus der Sicht von über 3000 befragten Personen in der Schweiz einen grundlegenden Satz der Pastoralinstruktion „Communio et progressio“ (Nr. 138) über die Instrumente der Sozialen Kommunikation:

„Die katholische Presse öffnet sich der ganzen Welt. Sie bringt Nachrichten, Kommentare und Meinungen über alle Aspekte des heutigen Lebens; siegreift alle Schwierigkeiten und Probleme auf, mit denen der Mensch heute konfrontiert ist; all dies aber im Lichte christlicher Lebensauffassung. Sie soll zugleich Spiegel sein, der die Welt reflektiert, und im Licht, das ihr den Weg zeigt. Sie soll Forum für Begegnung und Gedanken. austausch sein."

Christliche Presseerzeugnisse in säkularem Umfeld sollen weder Führungsinstrumente, Erziehungsinstitutionen noch eine Kanzel außerhalb der Kirche sein. Das Publikum versagt einer derartigen Tendenzpresse schlichtweg die Gefolgschaft. Es sucht objektive, glaubwürdige und sachliche Informationen, die seinen vielseitigen Interessen entsprechen und eine eigene Meinungsbildung erlauben. Das Publikum erwartet zudem von der christlichen Presse, daß sie wahr, seriös, sozial und solidarisch sei, und damit liegt das Publikum nochmals klar auf der Linie von „Communio et progressio“ ( $\mathrm{Nr}$. 17):

„Jede Kommunikation muß unter dem obersten Gesetz der Aufrichtigkeit, Zuverlässigkeit und Wahrheit stehen. "Die Ansprüche christlicher Leser an ihre Presse schlieBen kritische Beiträge nicht aus; sie verlangen einfach eine klare Linie und ein hohes journalistisches Ethos. Die säkularen Medien ihrerseits zeigen einen geringen Bedarf an religiösen Themen, aber sie sind äußerst interessiert an der Einstellung der katholischen Kirche zu Themen unserer Zeit. Im Sinne des 'agenda-settings' dürfte dies eine ausgesprochene Chance für katholische Agenturen und Pressestellen sein: säkulare Themen aus christlicher Sicht! Im Pluralismus der konkurrienden Meinung der Gegenwart ist die Stimme der katholischen Kirche eine durchaus gefragte Stimme.

Im Hinblick auf das Äußere der katholischen, resp. christlichen Presse, unter besonderer Berücksichtigung der Jugendpresse, hat die Pastoralinstruktion „Communio et progressio" nochmals recht $(\mathrm{Nrn} .130+131)$ : 
„Die heutigen Menschen sind von den Kommunikationsmitteln so sehr an perfekte Darstellung und gewinnenden Stil gewöhnt, daß sie niedriges Niveau bei öffentlichen Veranstaltungen kaum noch hinnehmen, schon gar nicht bei solchen mit religiösem Charakter wie liturgische Feiern, Predigten oder christliche Unterweisung. ... Will man die übliche Verkündigung und christliche Unterweisung interessant und wirksam gestalten, wird man sich, soweit es möglich ist, der Kommunikationsmittel bedienen und sich überhaupt in Sprache und Darstellungsweise der modernen gesellschaftlichen Kommunikation anpassen. " Der christlichen Presse in säkularem Umfeld stehen also klare Erwartungen von seiten des Publikums gegenüber: umfassende, objektive, glaubwürdige, sachliche Information; klare Beiträge zur persönlichen Meinungsbildung; Hinweise zur Einstellung der katholischen Kirche zu Themen und Problemen unserer Zeit; attraktive Präsentation und Sprache.

\section{Anmerkungen}

Othmar Baeriswyl; Louis Bosshart: Der Markt für die christliche Meinungspresse, Ergebnisse einer Umfrage bei Lesern des „Vaterlandes“, der „Ostschweiz“, des „Aargauer Volksblattes“ und der Nordschweiz, Freiburg: Arbeitsgemeinschaft der Katholischen Presse 1988

Louis Bosshart: Freiburger Nachrichten. Ergebnisse der Leserschaftsforschung, Freiburg 1988 [Manuskript]

Karin Ackermann: Die Massenmedien im Leben der Jugendlichen. Die „Freiburger Nachrichten " und ihre jungen Leser, Freiburg 1988 [Diplomarbeit]

Verena Colsman: Beurteilung des Angebots der Katholischen Internationalen Presseagentur (KIPA), Freiburg 1987 [Diplomarbeit]

Pascale Zbinden: Bekanntheitsgrad und Image der Internationalen Katholischen Presseagentur (KIPA), Freiburg 1997 [Diplomarbeit]

Markus Voegtlin: KIPA: eine Produkt-Analyse, Freiburg 1988 [Diplomarbeit]

\section{Summary}

Research carried out among young and adult readers of the Catholic youth press and the Christian opinion press in Switzerland shows that there are clear areas of expectation with regard to what the different media offer. The young look for information about teenage sub-culture, leisure activities and environment problems and for advice on their personal problems (sexuality, relationships etc.). Adults prefer themes that are by and large „workly“. They look for information on current political affairs and for ideas about personal fulfilment in life. Neither young people nor adults look primarily for religious themes in their media. But they value clear. Christian principles. Really important news in the Catholic Church is also looked for, in particular information on secular themes from a Christian viewpoint (especially in the secular media). The notion that the mass media are a second pulpit cannot be upheld in a market situation of this kind.

\section{Résumé}

Des sondages parmi les lecteurs adolescents et adultes de la presse catholique pour la jeunesse et la presse d'opinion chrétienne en Suisse révèlent des attentes distinctes en ce qui concerne les offres de différents mass-media. Les jeunes cherchent des informations sur la culture parallèle des jeunes, les loisirs, les problèmes écologiques, ainsi que des conseils sur des problèmes personnels (sexualité, rapports). Les préférences des destinataires adultes sont "laiques" dans une large mesure. Ils cherchent des informations sur la vie quotiedienne politique et la manière personelle de vivre. $\mathrm{Ni}$ des segements du public adolescent ni adulte ne se renseignent de sujets premier lieu religieux dans leurs média. Cependant, ils tiennent è une nette base chrétienne. Des actualités vraiment importantes de l'Eglise catholique sont aussi demandées, particulièremnt - et cela concerne d'abord les mass-média laics - des informaticns sur des thèmes séculiers d'un point de vue ecclesiastique. Une conception des mass-média comme une deuxième chaire est vouée è l'èchec dans cette situation du marché. 


\section{Resumen}

Investigaciones entre lectores jóvenes y adultos de las ediciones juveniles cristianas, muestran categorias de expectativas determinadas, referente a las ofertas de varios medios. Los jóvenes buscan informaciones sobre la subcultura joven, sobre actividades de tiempo libre, sobre problemas del medio ambiente asi como consejos en cuanto a problemas personales (sexualidad, relaciones). Las preferncias de temas de los lectores adultos, son en gran parte "profanas“. Ellos buscan informaciones sobre los quehaceres politicos y sobre la vida personal. Ni los lectores jóvenes, ni los adultos se informan en sus medios de temas primordialmente religiosos. Sin embargo requieren un fundamento claramente cristiano. Tambien hay demanda por noticias realmente importantes en el area de la iglesia católica, especialmente - y esto sobre todo en medios profanos informaciones sobre temas profanos desde el punto de vista de la iglesia. La idea de un medio de masas igual al púlpito fallaria inevitablemente en tal situacion del mercado. 